\title{
Calculating method of optimal path of data of traffic network Sun- Hongfeng ${ }^{1, a}$, Li- Ying ${ }^{1, a}$, Yang-Yanchun ${ }^{1, a}$ \\ ${ }^{1}$ Information Technology School, Shandong Women's University, Jinan, 250030, China \\ a sunhongfeng_sd@163.com
}

Keywords: Traffic flow; Particle swarm algorithm; Dynamic programming; The optimization of path; Time dependent

\begin{abstract}
According to the influence of traffic flow change on the selection of vehicle routing, to find a solution to the planning of travel routes in the situation that knowing the change of traffic flow. By using the method of particle swarm algorithm combined with dynamic programming to optimize the path of the vehicle, we can get the excellent route of each vehicle under the influence of the traffic flow. Simulating based on the road network structure and traffic data in real environment, the results showing that this method can increase the authenticity and dynamic of path optimization, and the road traffic flow and vehicle travel time having important influence on vehicle routing choice.
\end{abstract}

\section{Introduction}

Route planning of vehicle based on traffic flow makes the change of road network tend to diversity, authenticity. The optimization of path is not only to optimize the service order among customer points but also to optimize the route among the customer points based on the real environment. The target of optimization is from the shortest distance to the shortest time. In modern society time has gradually become the most important asset in people's live, the using efficiency of time becoming a prerequisite for pursuing maximum benefits of all walks of life. In the logistics industry, with the increase of the cost of logistics, how to timely and efficiently to complete the transport task has become an urgent problem of the logistics business enterprise to solve. Particle swarm optimization algorithm has merits of less number of individuals, simple operation, fast convergence speed, easy to realize, having been widely used by scholars in the field of computer science and management science, and having achieved many results. Wu Kaijun et al using binary particle swarm algorithm to optimize the vehicle routing, Zhang Liyan Zhang Wenjing proposed the application of particle swarm algorithm in the optimization of vehicle routing. In this paper, using particle swarm algorithm with the adaptive weight to optimize the initial of customer points and vehicle, optimizing the routine among the customer points phased according to the changes of the traffic flow of each road based on this, finally got the optimal route of distribution of delivery vehicles.

\section{Calculating method of optimal path of data of traffic network}

The problem of vehicle studied in this paper is ${ }^{m}$ vehicles to dispatch goods to customers successively. If the customer point is not scheduled to arrive at the destination, using the time penalty measures to deal with it.

The objective function

$$
\begin{aligned}
& \min z=\sum_{m \in M} \sum_{k \in V_{m}}\left\{r \cdot d d\left(m, x_{1}\right)+\sum_{x_{j} \in C_{m}^{k}} d\left(x_{j-1}, x_{j}\right)+d\left(x_{\mid C_{m}^{k}} \mid, m\right)\right]+ \\
& {\left[\sum_{x_{j} \in C_{m}^{k}}\left[f \cdot \max \left(s t_{x_{i}}-t_{x_{i}}^{k}, 0\right)+f_{x_{i}} \cdot \max \left(t_{x_{i}}^{k}-e t_{x_{i}}, 0\right)\right]\right\}+} \\
& \sum_{m, n \in M}\left[2 r \cdot d(m, n) \cdot \max \left(\left[\sum_{c \in C} y_{c m n} g_{C} / \omega\right],\left[\sum_{c \in C} y_{C m n} g_{C} / \omega\right]\right)\right]
\end{aligned}
$$




$$
\begin{aligned}
& \text { The constraint conditions } \\
& \text { st. } \quad V_{p} \cap V_{q}=\varnothing \quad\left(\left|V_{p} \cap V\right|_{q}=0\right) \quad \forall p, q \in M \\
& C_{1}^{1} \cup C_{1}^{2} \cup \cdots \cup C_{1}^{\left|V_{1}\right|} \cup \cdots \cup C_{|M|}^{1} \cup C_{|M|}^{2} \cup \cdots \cup C_{|M|}^{\left|V_{|M|}\right|}=C \\
& C_{p}^{u} \cap C_{p}^{v}=\varnothing, \quad \forall p, q \in M, \forall u \in V_{p}, \forall v \in V_{q} \\
& y_{x_{i}} \operatorname{center}\left(x_{i}\right) m=\left\{\begin{array}{l}
1, m \neq \operatorname{center}\left(x_{i}\right) \\
0, \text { others }
\end{array} \quad \forall m \in M, \quad k \in V_{m}, \forall x_{i} \in C_{m}^{k}\right. \\
& \operatorname{MinZ}=F_{\text {time }} \sum_{a \in s} \sum_{b \in s} \sum_{t=\alpha}^{\beta-1} x_{a b}^{t} T_{a b}^{t}+F_{w} \sum_{a \in s} w_{a}+F_{d} \sum_{a \in s} d_{a}
\end{aligned}
$$

The first part is the total time cost the vehicle in the above formula, the second part representing the total cost of vehicle travel distance, the second and third part said the cost of penalty due to vehicles not service on time. ${ }^{S}$ expresses the set of all customer points, ${ }^{p}$ saying the collection of the customer points and the path nodes, ${ }^{w_{s}}$ saying the waiting time in the customer point $a, d_{s}$ saying the delaying time in the customer point $a, F_{m}$ expressing travel cost of unit time, $F_{\mathrm{w}}$ being the waiting cost coefficient of the vehicle's early arriving, ${ }^{F_{d}}$ being the delay cost coefficient of the vehicle's late arriving, $T_{a s}$ said the time the vehicle in ${ }^{t}$ time from ${ }^{a}$ customer point to the customer $b, x_{a b}^{s}$ is the decision variable and its value is 1 , it saying the time the vehicle in $t$ time from $a$ customer point to the customer $b$.

The constraint conditions of the problem are as follows:

The constraints of vehicles in the departure time $t_{s}$ of customer point ${ }^{a}$ and the arrival time ${ }^{t_{s}}$, $\left[t_{s}, t_{s}\right]$ saying the time window of the plan period

$$
t_{a}^{e}=t_{a}+T_{a j}^{t} \quad t_{a} \in\left(t_{s}, t_{e}\right], t_{a}<t_{a}^{e} \leq \beta, a \in S, j \in P
$$

The calculation of waiting time and delay time of customer point ${ }^{a}$, ${ }^{p_{a}}$ says the expected service time of customer point $a, e_{a}$ saying the time arriving customer point $a$.

$$
\begin{array}{ll}
w_{a}=\operatorname{Max}\left(0, p_{a}-e_{a}\right) & , a \in S \\
d_{a}=\operatorname{Max}\left(0, e_{a}-p_{a}\right) & , a \in S
\end{array}
$$

The constraint of a car accessing the customer point $a$

$$
\sum_{\substack{t=a \\
j \in P \\
j \neq a}}^{w} x_{j a}^{t}-\sum_{\substack { t=a \\
\begin{subarray}{c}{i \in P \\
a \neq i{ t = a \\
\begin{subarray} { c } { i \in P \\
a \neq i } }\end{subarray}}^{w} x_{a i}^{t}=0, \quad a \in S
$$

The constraints of capacity, ${ }^{q_{a}}$ expresses the required amount of goods of the ${ }^{a}$ customer, $Q$ saying the rated load of vehicle, ${ }^{y_{a}^{k}}$ being a decision variable, when the vehicle $\mathrm{K}$ passing the customer $a$ its value being 1 , otherwise being 0 .

$$
\sum_{s \in=} q_{s} y_{s}^{k} \leq Q
$$

\section{The calculation of travel time between customers}

Calculating the travel time between customer points we should consider time, section and other factors. The departure time of customer point should be determined according to the departure time $t_{s}$ and the travel time $T_{s s}$ before the vehicle come into the customer point. The routine of vehicle in the customer or customer points or customer point to the distribution center can use searching method of rolling at both ends to determine the section the routine including, the specific steps being as follows:

The first step: search all the nodes connected by arcs with the customer (distribution center) ${ }^{a}$, 
expressed by set $v_{s}$

The second step: search all the nodes connected by arcs with the customer (distribution center) ${ }^{b}$, expressed by set $v_{b}$

The third step: comparing $v_{s}$ and $v_{b}$ to find the common point, if there is a common point we can get the routine between customer ${ }^{a}$ and customer $^{b}$, reckoning in the route collection ${ }^{\mathrm{R}}$; If there is no common point, we continue to search taking the point of $v_{s}$ or $v_{b}$ as center, comparing the searched point with $v_{s}$ or $v_{b}$, in principle the less the number of nodes the better, after as little as possible, setting the number of nodes, being more than it ,the search stops.

According to the above calculation got the routine between customer ${ }^{a}$ and customer ${ }^{b}$, for each route finding out the section of road network ${ }^{E_{4}}$ contained in the route, ${ }^{i}$ and ${ }^{j}$ being the connected nodes of section of road network. ${ }^{t}$ said the time going into the section ${ }^{E_{\mu}}$, $q_{t_{y}}$ said the traffic flow at the time ${ }^{t_{y}}$, using the formula (4) (5) can calculate the average speed $v_{\mathrm{t}_{y}}$ of vehicles passing the section ${ }^{E_{4}}$. According to Cao Xiangyu [14] mentioned in the paper that using the queuing theory, intersection delay and other methods to calculate travel time, here in order to simplify the calculation of ignore the impact of intersection delay on the travel time, getting the computational formula of travel time of section ${ }^{E_{i j}}$ :

$$
T_{t_{i j}}=\frac{L_{i j}}{v_{t_{j j}}} \quad(7) \quad v_{t_{i j}}=\frac{a_{1} v_{0}}{1+\left(q_{t_{i j}} / c\right)^{\beta}}
$$

In the formula, ${ }^{L_{y}}$ saying the length of section ${ }^{E_{\bar{y}}}$, the hypothesis of $v$ and ${ }^{c}$ of each section is consistent. Only considering the influence of the change of traffic flow with time and position change of the road, according to the gotten travel time to calculate time cost to find out the routine with least time cost.

\section{Description of the algorithm}

\section{The particle encoding method in vehicle routing problem}

This paper uses for reference of literature [15], constructing a $2 M$ dimensional space corresponding to have VRP problem with $M$ task points, the $2 M$ dimension vector $X$ corresponding to each particle is divided into two dimensional vector: ${ }^{X_{\text {i }}}$ (the corresponding vehicle of each task ) and $X_{i r}$ (priority of transporting path of corresponding vehicle of each task). For example, one has 7 customers, 3 cars having vehicle routing problem, the position vector $X$ of the ${ }^{i}$ particle being:

Table 1 The vector of a particle

\begin{tabular}{llllllll}
\hline $\begin{array}{l}\text { Customer } \\
\text { points }\end{array}$ & 1 & 2 & 3 & 4 & 5 & 6 & 7 \\
\hline$X_{i v}$ & 1.7 & 2.3 & 2.6 & 3.4 & 3.9 & 1.2 & 3.6 \\
\hline$X_{i r}$ & 0.9 & 2.8 & 3.7 & 1.4 & 2.6 & 4.4 & 1.8 \\
\hline
\end{tabular}

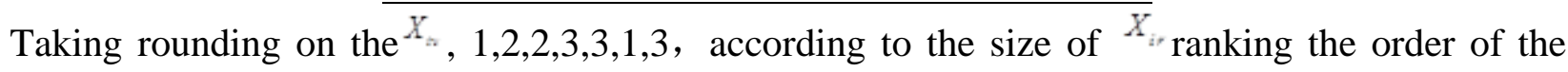
customer to get the task allocation of vehicle and customer service order (No. 0 expresses distribution center)

Vehicle 1:0-1-6-0 Vehicle 2:0-2-3-0 Vehicle 3:0-4-7-5-0

\section{The description of algorithms process}

The first step: The initialization of algorithm. Inputting the corresponding parameters of network, the determination of particle size ${ }^{n}$, the inertia factor ${ }^{\omega}$, learning factor ${ }^{c_{1}} 、 c_{2}$, maximum number of iterations ${ }^{N}$.

The second step: initializing particle swarm, the vector $x_{*}$ of each particle random chooses the 
real number between $1 \sim K$ (vehicles), each dimension of $x_{\text {random choosing the real number }}$ between $1 \sim L$ (the number of tasks); each dimension of each velocity vector $V_{*}$ choosing the real number between ${ }^{-(K-1) \sim(K-1)}$ (the number of vehicle), each dimension of $V_{s}$ random choosing the real number between $-(L-1) \sim(L-1)$. The boundary value of the range of the corresponding is the boundary value of particle activity.

The sixth step: determine whether the maximum number of iterations reached the preset maximum number of times, if reached, then stopped the iteration, outputting of the optimal solution gbest, otherwise go to the third step.

\section{Simulation experiment}

\section{Description of the experiment and parameter setting}

The experimental environment: lenovo corei5,2G memory, WindowsXP,MATLAB17.0.

In this experiment, according to the road network of the city, intercepting 105 nodes, 164 road network of sections, according to the actual operation situation of vehicle of network using the simulation tool Netlogo to simulate the change of traffic flow of every section, getting the basic data value of traffic flow of each section. Then take the experiment with MATLAB on the obtained data base, the experiment with 8 customers, 3 cars to optimize the distribution, the maximum load of each car being 8t, customer data as shown in table 1 .

Table 2 customer data

\begin{tabular}{clll}
\hline $\begin{array}{c}\text { Customer } \\
\text { point } i\end{array}$ & $q_{i}$ & $p_{i}$ & Coordinate \\
1 & 2 & 33 & {$[40,21]$} \\
2 & 3 & 60 & {$[24,43]$} \\
3 & 3.5 & 110 & {$[35,67]$} \\
4 & 2.5 & 72 & {$[60,48]$} \\
5 & 4 & 119 & {$[78,50]$} \\
6 & 1.5 & 44 & {$[60,30]$} \\
7 & 3 & 123 & {$[83,70]$} \\
8 & 4.5 & 73 & {$[80,35]$} \\
\hline
\end{tabular}

All the vehicles starting from the distribution center, the rate of change of vehicle of the vehicle speed determined according to the need of the departure time and the after road from table 2. The penalty factor that not according to the expected arrival time to reach customers is ${ }^{F_{w}}=F_{d}=10$, the unit cost of travel time being $F_{m=5}=5$, the number of nodes being $\varepsilon=7$. The parameters of particle swarm algorithm is the swarm $\operatorname{size}^{n}=50$, the dimension of particle being ${ }^{D}=16$, the number of iterations being $N_{\mathrm{max}}=50$, repeating the experiment for 10 times.

\section{Experimental results}

The corresponding path and related parameters of ${ }^{t_{1}}$ time, the brackets to express the time of reaching the customers or through the sections:

In this example using that the departure time changing and route being constant to compare the travel time of the vehicle, on the other hand, using the same starting time to compare the influence of different starting line on travel time, this kind of comparing control method can enhance the comparability and readability of the results.

Through a lot of experiments we can find that the influence of the traffic flow on the vehicle path planning, mainly reflecting in when the starting time different, the same purpose of vehicle transportation, through different routes, which can clearly see the different route of the both, and the vehicle reaches the accuracy of customer is different in different departure time. In the actual transportation environment, traffic flow path has a great influence on the selection and planning of vehicle. 


\section{Conclusion}

This paper proposed the map of road network based on the information of traffic flow, and based on this to take path planning. After the introduction of traffic flow, we should consider the effect of traffic flow on the average velocity of section, traffic flow in a day being changing. This paper has two innovation points: one is that the path planning considering the impact of traffic flow on the path selection and path planning, being different from the past path planning of vehicle in a static environment; two is the service optimization on customers, focuses on the selection of path between customers. Optimization of customers through two aspects: on the one hand, the optimization of customer order; on the one hand, to optimize the route selection between the customer. In this paper, using the particle swarm algorithm to plan the customer service order, using the dynamic programming method to plan the route between customers, through the experiment, using the change of traffic flow to take path planning can reduce the transport time, improve the timeliness of delivery and reduce the transportation cost.

\section{Acknowledgement}

Natural science foundation of Shandong Province: Statistical Analysis of the Massive Traffic Data Based on Cloud Platform, Project No: ZR2013FL026

University science and technology projects in Shandong Province: Real Time Data Processing Method massive traffic moving under cloud, Project No: J14LN01

\section{References}

[1] Jie He, Yishuang Geng and Kaveh Pahlavan, Toward Accurate Human Tracking: Modelling Time-of-Arrival for Wireless Wearable Sensors in Multipath Environment, IEEE Sensor Journal, 14(11), 3996-4006, Nov. 2014

[2] Lv, Zhihan, Liangbing Feng, Haibo Li, and Shengzhong Feng. "Hand-free motion interaction on Google Glass." In SIGGRAPH Asia 2014 Mobile Graphics and Interactive Applications, p. 21. ACM, 2014.

[3] Zhong, Chen, Stefan Müller Arisona, Xianfeng Huang, Michael Batty, and Gerhard Schmitt. "Detecting the dynamics of urban structure through spatial network analysis." International Journal of Geographical Information Science 28, no. 11 (2014): 2178-2199.

[4] Li, Wubin, Johan Tordsson, and Erik Elmroth. "An aspect-oriented approach to consistency-preserving caching and compression of web service response messages." In Web Services (ICWS), 2010 IEEE International Conference on, pp. 526-533. IEEE, 2010.

[5] Y. Geng, J. He, K. Pahlavan, Modeling the Effect of Human Body on TOA Based Indoor Human Tracking[J], International Journal of Wireless Information Networks 20(4), 306-317

[6] Lv, Zhihan, and Tianyun Su. "3D seabed modeling and visualization on ubiquitous context." In SIGGRAPH Asia 2014 Posters, p. 33. ACM, 2014. 